\title{
Low Mach-number collisionless electrostatic shocks and associated ion acceleration
}

\author{
I. Pusztai, ${ }^{1,}$ a) J. M. TenBarge, ${ }^{2,3,4}$ A. N. Csapó, ${ }^{1}$ J. Juno, ${ }^{2}$ A. Hakim, ${ }^{4}$ L. $\mathrm{Yi}^{1}{ }^{1}$ and \\ T. Fülöp ${ }^{1}$ \\ 1) Department of Physics, Chalmers University of Technology, SE-41296 Göteborg, \\ Sweden \\ ${ }^{2)}$ Institute for Research in Electronics and Applied Physics, University of Maryland, \\ College Park, MD 20742, USA \\ 3) Department of Astrophysical Sciences, Princeton University, Princeton, NJ 08543, \\ $U S A$ \\ 4) Princeton Plasma Physics Laboratory, Princeton, NJ 08543, \\ $U S A$
}

(Dated: 21 December 2017)

The existence and properties of low Mach-number $(M \gtrsim 1)$ electrostatic collisionless shocks are investigated with a semi-analytical solution for the shock structure. We show that the properties of the shock obtained in the semi-analytical model can be well reproduced in fully kinetic Eulerian Vlasov-Poisson simulations, where the shock is generated by the decay of an initial density discontinuity. Using this semi-analytical model, we study the effect of electron-to-ion temperature ratio and presence of impurities on both the maximum shock potential and Mach number. We find that even a small amount of impurities can influence the shock properties significantly, including the reflected light ion fraction, which can change several orders of magnitude. Electrostatic shocks in heavy ion plasmas reflect most of the hydrogen impurity ions.

Keywords: collisionless shock, ion acceleration, laser plasma

\section{INTRODUCTION}

Collisionless shocks are common in space, astrophysical and laboratory plasmas, and their efficiency as particle accelerators is well established 112. In the context of laser-produced plasmas, collisionless shocks may be used for ion acceleration. When a laser hits an overdense plasma, it leads to electron heating and density steepening. This scenario can result in a collisionless shock, which propagates into the target. Ions can be reflected off the moving electrostatic potential front with twice the shock velocity in the rest frame of the upstream population. Recent experimental and numerical results have shown that monoenergetic acceleration of protons can be achieved at modest laser intensities, albeit with rather low laser-to-particle energy conversion efficiency $\stackrel{3}{3}$. High energy ions with narrow energy-spread would be very attractive for a wide range of applications.

In particular, non-relativistic electrostatic collisionless shocks have been observed both in the laboratory ${ }^{6}$ and in space ${ }^{9}$ and can also be of interest in laser-plasma driven shock acceleration of protons. The theoretical basis for electrostatic, ion-acoustic-type shocks were laid out already in the $1960 \sqrt{10}$, and it was realized that such shocks can be sustained by the reflection of ions on the shock potential11. Numerical studies of collisionless shocks $4\left[12, \frac{15}{4}\right.$ have mostly used Particle-In-Cell16 algorithms. Eulerian Vlasov-Maxwell approaches avoid issues with particle statistics; this difference can be particularly important for the accurate modeling of scenarios where the reflected ion fraction is small, or where turbulent fluctuations shape the shock dynamics 17 . However, there are only a limited number of electrostatic shock studies using Vlasov-Maxwell tools $5 \mid 17$.

\footnotetext{
a) Electronic mail: pusztai@chalmers.se
} 
Analytical models are appealing due to their simplicity and can be useful for gaining physical understanding as a complement to more extensive numerical simulations. Analytical models of various sophistication range from cold ion fluid approaches ${ }^{\sqrt{11}}$ to exact kinetic shock solutions 5 . A simple treatment of the non-magnetized collisionless shock structure, taking into account finite ion temperature, is given in Refs. 19] and 20. In the current paper, we adopt this formalism but with a more consistent treatment of the trapped regions in the ion phase space, to address the effects of the ion composition on shock properties and the reflected ion fraction in low Mach number (i.e., $M \approx 1-1.5$ ) electrostatic shocks.

First, through comparisons to fully kinetic simulations using the Eulerian Vlasov-Poisson solver contained in Gkeyli 21 , we demonstrate that the properties of the shock are well reproduced by the semi-analytical model. Then, we use the model to investigate the effect of a heavy ion component on the existence of the shock, the Mach number, and reflected ion fraction. We show that the effect of a heavy ion component can be important also if it is only present in small quantities, as it may affect the electrostatic potential and the shock propagation speed, and thereby have a strong influence on the ion spectrum. For instance, only a few percent of a carbon impurity increases the maximum electrostatic potential significantly for the same electron-to-ion temperature ratio and Mach number. This potential, in turn, affects the reflected ion fraction exponentially. Even the existence of shock solutions is affected by the impurity concentration.

We have also investigated shock properties in the case where the main ion component is a heavy ion and the trace impurity is hydrogen. This case is the typical scenario in laser-driven ion acceleration experiments, where solid aluminum foil target having hydrogen impurities on their surface is often used. In this case, the semi-analytical model predicts that the hydrogen ions are almost all reflected. This prediction is corroborated with Vlasov-Poisson simulations, showing excellent agreement both with the distribution functions and the shock speed.

The structure of the paper is the following. In Section [I] we describe the semi-analytical model and the assumptions behind it. Next, in Section III we compare the model to kinetic simulation results using a Vlasov-Poisson solver, and demonstrate the similarities of the ion and electron distribution function, the electrostatic potential and Mach number. In Section IV], we proceed to use the semi-analytical model to study the effect of ion composition and electron-to-ion-temperature ratio. Finally, we summarize and conclude in Section $\mathrm{V}$

\section{ELECTROSTATIC SHOCK}

Electrostatic shocks are sustained by an electric field that is linked to a density gradient between the downstream and upstream plasmas. We focus on the effect of ion composition on the shock properties, while we are not concerned about which physical process generated the shock, or about its long term stability. In the following, we describe a simple, onedimensional model for a collisionless electrostatic shock, which is sufficient for our purposes, and it is similar to the model of Ref. 19 .

The potential $\phi$ increases from zero in the far upstream region $(x \rightarrow \infty)$ to some positive value $\phi_{\max }$ at $x=0$, as illustrated in Fig. 11. In the downstream region, $x<0$, the electrostatic potential is finite and oscillatory. In the laboratory frame, the shock propagates in the $+x$ direction with a velocity $V$, whereas the ions sufficiently far ahead of the shock have a zero flow speed. We assume $V \sim c_{s} \gg v_{i}$, where $v_{i}=\sqrt{T_{i} / m_{i}}$ is the thermal speed of the main ions with mass $m_{i}$ and temperature $T_{i}$, and $c_{s}=\sqrt{Z_{i} T_{e} / m_{i}}$ is the sound speed, with $T_{e}$ the electron temperature and $Z_{i}$ the ion charge number. Thus, in the shock frame, the far upstream ion flow velocity is $-V$; these ions represent the incoming population in Fig. 1p. The phase-space separatrix between ions that can pass through the potential barrier and those reflected from it is represented by the dashed line in the $x>0$ and $v<0$ region of Fig. 1 $\mathrm{b}$. We consider only a certain vicinity near the shock, where the reflected ions are already present for $x>0$, and do not treat the problem of what happens with the leading edge of the reflected population (i.e., the so-called "foot" problem²2). 
(a)

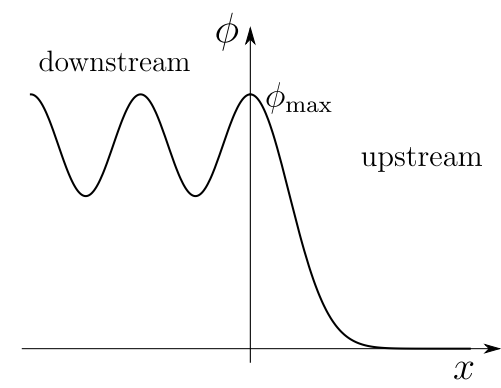

(b)

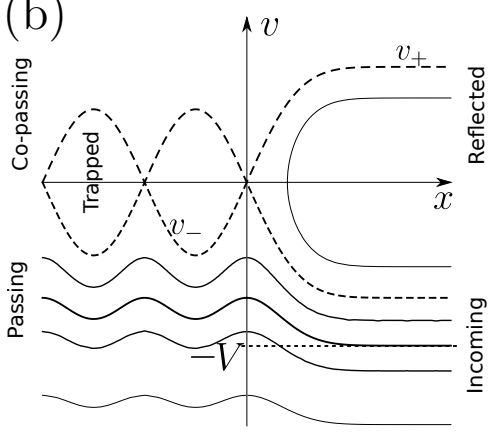

FIG. 1: (a) Electrostatic potential of the shock structure showing a monotonic increase from $\phi=0$ to $\phi_{\max }$ in the upstream region, and an oscillatory behavior with $0<\phi \leq \phi_{\max }$ in the downstream region. (b) Phase-space plot of the ion distribution showing the different populations (incoming, reflected, passing). In the upstream region the shock potential reflects a fraction of the ions, while in the downstream the density of passing ions is oscillatory in $x$.

We use a notation and normalizations that accommodate multiple ion species with arbitrary charge and mass. The "bulk" ion species that dominates the dynamics will be denoted by index $i$, and the impurities by index $z$, while $e$ refers to electrons and $j=\{i, z\}$ to ions in general. For any physical quantity $X$, we define a dimensionless normalized quantity $\hat{X}=X / \bar{X}$, with $\bar{X}$ a species-independent normalizing quantity. In particular $\bar{T}=T_{i}$ is the bulk ion temperature, $\bar{n}=n_{0}$ is the far upstream incoming bulk ion density, $\bar{\phi}=T_{i} / e$, $\bar{v}=\sqrt{T_{i} / m_{p}}$ with the proton mass $m_{p}, \bar{x}=\left[T_{i} \epsilon_{0} /\left(e^{2} n_{0}\right)\right]^{1 / 2}$ (which is $\bar{x}=\bar{v} / \omega_{p p}$, with $\omega_{p p}$ the proton plasma frequency at $\left.n_{0}\right)$. Finally, distribution functions are normalized by $\vec{f}=\bar{n} / \bar{v}$.

The general solution of the collisionless, steady state ion kinetic equation

$$
v \frac{\partial f_{j}}{\partial x}-\frac{Z_{j} e}{m_{j}} \frac{d \phi}{d x} \frac{\partial f_{j}}{\partial v}=0
$$

is $f_{j}=f_{j}\left(E_{j}\right)$, with $E_{j}=m_{j} v^{2} / 2+Z_{j} e \phi$ the total energy. Thus, the distribution prescribed at the boundaries where particles are incoming into our domain $(v<0$ at $x \rightarrow \infty$ and $v>0$ at $x \rightarrow-\infty)$ simply needs to be extended along the contours of constant total energy. Such lines are shown by solid lines in Fig. 1 $\mathrm{b}$, which then represent contours of the distribution function, with the thick line corresponding to the maximum of the distribution. We take the far upstream ion distribution to be a Maxwellian, $f_{j}^{(+\infty)}=$ $n_{j} /\left(v_{j} \sqrt{2 \pi}\right) \exp \left[-(v+V)^{2} /\left(2 v_{j}^{2}\right)\right]$, for $x \rightarrow \infty$ and $v<0$. We are also free to specify the ion distribution in the trapped regions of phase-space (see Fig. 1 1 ); we assume that the trapped regions are empty. Furthermore, consistent with the assumption $V>v_{i}$, we neglect the small co-passing population, appearing above the trapped and the reflected regions, as it would represent a tail of a Maxwellian and its contribution to a charge imbalance between upstream and downstream is negligible.

Thus, the normalized ion distribution function and the ion density are given by

$$
\begin{aligned}
& \hat{n}_{j}^{ \pm}(\hat{x})=\int_{-\infty}^{\hat{v}_{ \pm}} \hat{f}_{j} d \hat{v} \equiv \frac{\hat{n}_{j}}{\sqrt{2 \pi \hat{T}_{j} / \hat{m}_{j}}} \\
& \times \int_{-\infty}^{\hat{v}_{ \pm}} d \hat{v} \exp \left\{-\frac{\left(\sqrt{\hat{v}^{2}+2 Z_{j} \hat{\phi} / \hat{m}_{j}}-\hat{V}\right)^{2}}{2 \hat{T}_{j} / \hat{m}_{j}}\right\},
\end{aligned}
$$


where the + and - upper indices refer to upstream and downstream, respectively. Above the velocity separatrices given by $\hat{v}_{ \pm}= \pm \sqrt{2\left(Z_{j} / \hat{m}_{j}\right)\left(\hat{\phi}_{\max }-\hat{\phi}\right)}$, the distribution function vanishes: $\hat{f}_{j}\left(\hat{v}>\hat{v}_{ \pm}\right)=0$ for $\pm \hat{x}>0$. In the far upstream region (i.e., where the reflected population is present, but $\hat{\phi}=0$ ) the density can be explicitly evaluated to

$$
\hat{n}_{j}^{+}(+\infty)=\frac{\hat{n}_{j}}{2}\left[1+2 \operatorname{erf}\left(\tilde{V}_{j}\right)+\operatorname{erf}\left(\sqrt{\Psi_{j}}-\tilde{V}_{j}\right)\right]
$$

where $\tilde{V}_{j}=\hat{V} / \sqrt{2 \hat{T}_{j} / \hat{m}_{j}}, \Psi_{j}=Z_{j} \hat{\phi}_{\max } / \hat{T}_{j}$, and erf denotes the Gauss error function.

The ion density in Eq. (2) is similar to the one used in Ref. 19, except that there the distribution function appears to be extended up to $\hat{v}=0$ in the downstream region, even when $\hat{\phi}<\hat{\phi}_{\max }$. This assumption is not consistent with the constancy of the distribution along the energy contours in a steady state. Although the incorrect treatment does not effect $\hat{\phi}_{\max }$, because that depends on the upstream distribution function, it increases the amplitude and the wavelength of the oscillation of $\hat{\phi}$ in the downstream region.

The reflected ion fraction can also be calculated from the ion distribution function:

$$
\begin{aligned}
\alpha_{j} & =\frac{\int_{0}^{\sqrt{2 \hat{\phi}_{\max } Z_{j} / \hat{m}_{j}}} d \hat{v} \exp \left\{-\frac{(\hat{v}-\hat{V})^{2}}{2 \hat{T}_{j} / \hat{m}_{j}}\right\}}{\int_{-\infty}^{0} d \hat{v} \exp \left\{-\frac{(\hat{v}+\hat{V})^{2}}{2 \hat{T}_{j} / \hat{m}_{j}}\right\}} \\
& =\left[\operatorname{erf}\left(\tilde{V}_{j}\right)+\operatorname{erf}\left(\sqrt{\Psi_{j}}-\tilde{V}_{j}\right)\right] /\left[1+\operatorname{erf}\left(\tilde{V}_{j}\right)\right] .
\end{aligned}
$$

Electron distribution functions assuming adiabatic trapping throughout the $m_{e} v^{2} / 2<$ $e \phi_{\max }$ region of phase space have been considered previously 23|24. Even though trapping can occur in the cases that we will consider, it is restricted to the regions bounded by the local potential minima of the downstream oscillation: $m_{e} v^{2} /(2 e)<\phi_{\max }-\phi_{\min ,-1}$, where $\phi_{\min ,-1}$ denotes a local potential minimum. Note, that the electrons that are not trapped in these downstream oscillations, but are merely constrained to the semi-infinite downstream region (i.e., those with $\phi_{\max }-\phi_{\min ,-1}<m_{e} v^{2} /(2 e)<\phi_{\max }$ ), have an infinitely long bounce time (unlike trapped particles considered in Ref. 25), and as such, they do not behave as real trapped particles. For simplicity, we will neglect a possible flattening of the distribution in the real trapped regions and consider a Maxwell-Boltzmann electron distribution $f_{e}=n_{e} /\left(v_{e} \sqrt{2 \pi}\right) \exp \left[-v^{2} /\left(2 v_{e}^{2}\right)+e \phi / T_{e}\right]$, where $v_{e}=\sqrt{T_{e} / m_{e}}$ is the electron thermal speed, and the constant $n_{e}$ is the far upstream electron density. As the flow speed $V / v_{e}$ is small in the limit $\sqrt{m_{e} / m_{i}} \ll 1$, the flow of the electron distribution in the shock frame is neglected. Thus, the normalized electron density is $\hat{n}_{e}(\hat{x})=\hat{n}_{e} e^{\hat{\phi} / \tau}$, where $\tau=T_{e} / T_{i}\left(=\hat{T}_{e}\right)$. To obtain the constant $\hat{n}_{e}$ we may assume that sufficiently far upstream the plasma is quasineutral. Thus the electron density is

$$
\hat{n}_{e}(x)=\sum_{j} Z_{j} \hat{n}_{j}^{+}(+\infty) e^{\hat{\phi} / \tau}
$$

where $\hat{n}_{j}^{+}(+\infty)$ is given by Eq. 3 .

Finally, the ion and electron densities can be used together with Poisson's equation to find the electrostatic field. In normalized quantities, Poisson's equation reads

$$
\frac{d^{2} \hat{\phi}(\hat{x})}{d \hat{x}^{2}}=\hat{n}_{e}(\hat{x})-\sum_{j} Z_{j} \hat{n}_{j}(\hat{x}) .
$$

Following the classic treatment ${ }^{1}$, Poisson's equation can be rewritten in terms of a Sagdeev potential $\Phi\left(\hat{\phi}, \hat{\phi}_{\max }\right)=\int_{0}^{\hat{\phi}}\left[\sum_{j} Z_{j} \hat{n}_{j}\left(\hat{\phi}^{\prime}, \hat{\phi}_{\max }\right)-\hat{n}_{e}\left(\hat{\phi}^{\prime}, \hat{\phi}_{\max }\right)\right] d \hat{\phi}^{\prime}$ so that we have 
$d^{2} \hat{\phi} / d \hat{x}^{2}=-\partial \Phi / \partial \hat{\phi}$ that, after multiplication by $d \hat{\phi} / d \hat{x}$, gives the familiar equation

$$
\frac{1}{2}\left(\frac{d \hat{\phi}}{d \hat{x}}\right)^{2}=-\Phi,
$$

analogous to the equation of motion of a particle in a potential. The condition $\Phi\left(\hat{\phi}_{\max }, \hat{\phi}_{\max }\right)=$ 0 determines the quantity $\hat{\phi}_{\max }$. A solitary wave occurs when the Sagdeev potential $\Phi$ has a local maximum at the origin $(\hat{\phi}=0)$ and goes through zero again at some finite value of $\hat{\phi}$. Shock-like structures can form when there is damping in the system, for example ion-reflection, which produces an asymmetry between upstream and downstream sides.

For a laser with moderately high intensity, the electron temperature can be expected to be in the $\mathrm{MeV}$ range ${ }^{4 / 26}$, and $\tau$ can range from a few tens to several hundred. Although the dispersion relation of sound waves in multi-species plasmas can be rather complex ${ }^{27}$, for simplicity, we define the Mach number $M=V / c_{s}=\hat{V} \sqrt{\hat{m}_{i} /\left(\tau Z_{i}\right)}$, with respect to the speed $c_{s}=\sqrt{Z_{i} T_{e} / m_{i}}$.

In the model used here - similarly to Ref. [19- both $\tau$ and $\hat{V}$ (or equivalently the Mach number $M$ ) are treated as inputs. For a given $\tau$ there can be a finite range of Mach numbers where shock solutions exist. Towards the highest Mach number of such a range, the amplitude of the downstream oscillation approaches zero (with decreasing wave length). The degenerate case of the amplitude becoming zero corresponds to a monotonic shock structure. The boundary condition $\partial \Phi / \partial \hat{\phi}=0$ at $\hat{\phi}=\hat{\phi}_{\max }$ produces such monotonic shock solutions. Representing an additional constraint, this boundary condition removes one degree of freedom, thus it can be used to calculate $\hat{V}$ for a given $\tau$, as done in Ref. 28,

\section{COMPARISON TO KINETIC SIMULATIONS}

We have performed simulations with the Gkeyl1 ${ }^{21}$ Vlasov-Poisson solver and compared the results to the analytical model presented in the previous section. The simulations evolve kinetic ion and electron species, starting from a density step as an initial condition. We focus on the electrostatic shock that develops at the initial density discontinuity and propagates into the low density region. For the comparisons, we use $1 \mathrm{x} 1 \mathrm{v}$ (one spatial and one velocity dimension), noting that increasing the dimensionality to $2 \times 2 \mathrm{v}$ leads to identical results ${ }^{1}$

The possible range of Mach numbers where shock solutions exist in our model increases with the temperature ratio $\tau$. In particular, below a certain $\tau$ value, no solutions exist. Although our ion distribution function is slightly different from that of Ref. 20, in a single species plasma we obtain a similar result for the allowed Mach number range to their Figure 2. The reason for this insensitivity to the exact form of the distribution is the following. At the upper Mach number threshold, where the monotonic shocks appear, $\hat{v}_{-}=$ 0 thus the two types of distribution function coincide. At the lower Mach number threshold, $\hat{\phi}_{\max }$ (determined by upstream dynamics) goes to zero, and the reflected fraction diminishes, as we will show. When this happens, the population above $\hat{v}_{-}$that distinguishes the two distribution functions, becomes negligibly small, and thus does not affect quasineutrality.

Therefore, we find it useful to choose a moderate value of $\tau$ with a wide range of possible solutions. This choice is convenient, since unlike in the analytical model, where the inputs are $\hat{V}$ and $\tau$, and $\hat{\phi}_{\max }$ is an output, in the simulation the inputs are the ratio of densities in the density discontinuity, and $\tau$. In this case, the density ratio determines the total potential drop across the whole simulation domain - as the electron distribution is close to

1 This behavior is expected, as in the absence of initial magnetic perturbations, a $1 \mathrm{x} 1 \mathrm{v}$ problem remains $1 \mathrm{x} 1 \mathrm{v}$ in continuum Vlasov simulations, since no coupling occurs to other velocity dimensions. This behavior is unlike PIC codes, where the statistical noise can provide a seed to various instabilities $29 \sqrt[31]{31}$ that can potentially break the initial symmetry of the solution. However, we note that for the setup studied here, no growth of instabilities was observed in a 2x3v PIC simulation either, using the EPOCH $\operatorname{code}{ }^{32}$; in particular, the noise in the magnetic field stayed at a constant level throughout the simulation. 


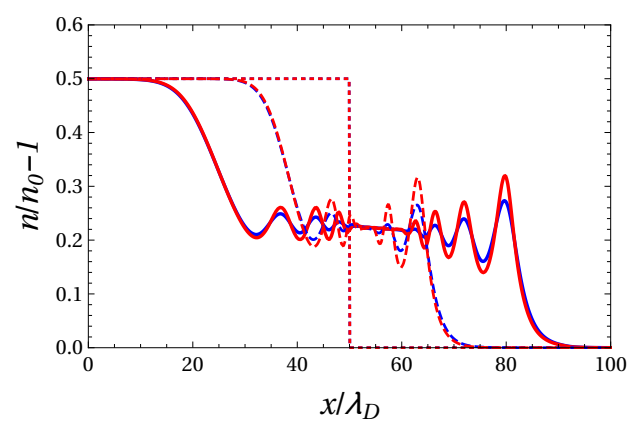

FIG. 2: Relative density difference from the far upstream value. Red: ion; blue: electron. Dotted lines: $t \omega_{p p}=0$, dashed lines: $t \omega_{p p}=15$, thick solid lines: $t \omega_{p p}=30$.

Maxwell-Boltzmann - and the potential drop across the shock (i.e., $\hat{\phi}_{\max }$ ) is approximately half of the total potential drop. By adjusting the density ratio, the shock potential and the shock propagation speed can be adjusted.

In the following comparison we assume $\tau=45$, an initial density ratio $n_{i}(x=-\infty) / n_{0}=$ 1.5 , and $v_{e}=0.1 c$. Both species are kinetic, with a mass ratio $m_{i} / m_{e}=1836$, the initial discontinuity in the density is located in the middle of the $L_{x}=100 \lambda_{D}$ wide simulation domain that has 768 grid points $\left(\lambda_{D}=\sqrt{\epsilon_{0} T_{e} /\left(e^{2} n_{e}\right)}\right.$ is the Debye-length). The range of electron velocities is $\left[-6 v_{e}, 6 v_{e}\right]$ and for ions it is $\left[-6 v_{i}, 12 v_{i}\right]$. The number of cells is 256 and 64 in configuration space and velocity space, respectively, with polynomial order 2 . The boundary conditions are open in configuration space and zero flux in velocity.

The relative density difference from the far upstream value $\left(n_{0}\right)$ for both electrons (blue curves) and ions (red curves) is shown in Fig. 2 at $t \omega_{p p}=\{0,15,30\}$. There are features of the shock in the simulation, related to the initial condition, which are not present in the analytical model: the downstream oscillatory part of the solution decays away from the shock front, similarly to the ion acoustic front structures found analytically in Ref. 33 for small density discontinuity initial conditions. Further downstream, the solution transitions into another oscillation, which leads up to the rarefaction front propagating in the opposite direction. Also, considering the distance between the initial density step location $\left(x / \lambda_{D}=\right.$ 50 ) and the maximum density point in the shock at $t \omega_{p p}=15$ and 30 we find that the propagation speed is increasing with time. Nevertheless, some properties of the shock front become approximately independent of the initial condition during the simulation. In particular the maximum value of the shock densities and their shape in the upstream region do not change significantly between $t \omega_{p p}=15$ and $t \omega_{p p}=30$.

Let us compare the simulation result to the analytical model at $t \omega_{p p}=30$. Using the analytical model for $\tau=45$, we identified a $V$ that produces similar maximum ion and electron densities to those in the simulated shock front. The result shown in Fig. 3 a with solid lines correspond to $\hat{V}=7.64(M=1.139)$, with $\hat{\phi}_{\max }=10.96$. The simulation result is shown with dashed lines, and $\hat{n}_{e}\left(\hat{n}_{i}\right)$ corresponds to blue (red) curves. The propagation speed is slightly higher than the one estimated for the simulation $M=1.133 \pm 0.003$ from the movement of the highest density position, although as we mentioned, the propagation speed has an increasing trend during the simulation. We can see that the difference between the maximum $\hat{n}_{i}$ and $\hat{n}_{e}$ values is very well captured.

Next, we compare the upstream and downstream distribution functions. The simulated (dashed lines) and analytical (solid lines) distribution functions are shown in Fig. 3b, for $\hat{x}=15$ (upstream, red) and $\hat{x}=-15$ (downstream, blue), respectively. Since $\hat{\phi}>0$ at these locations, the flow velocity of the bulk ion distribution is above the far upstream value, $-\hat{V}$. Analytically, the velocity space is empty above $\hat{v}_{-}=-2.29$ in the downstream region, there is a reflected population with a cutoff at $\hat{v}_{+}=2.53$ in the upstream region. Similar cutoffs are also seen in the simulation; in particular, there is practically no ion population in the trapped region downstream, which supports our choice of ion ansatz. 

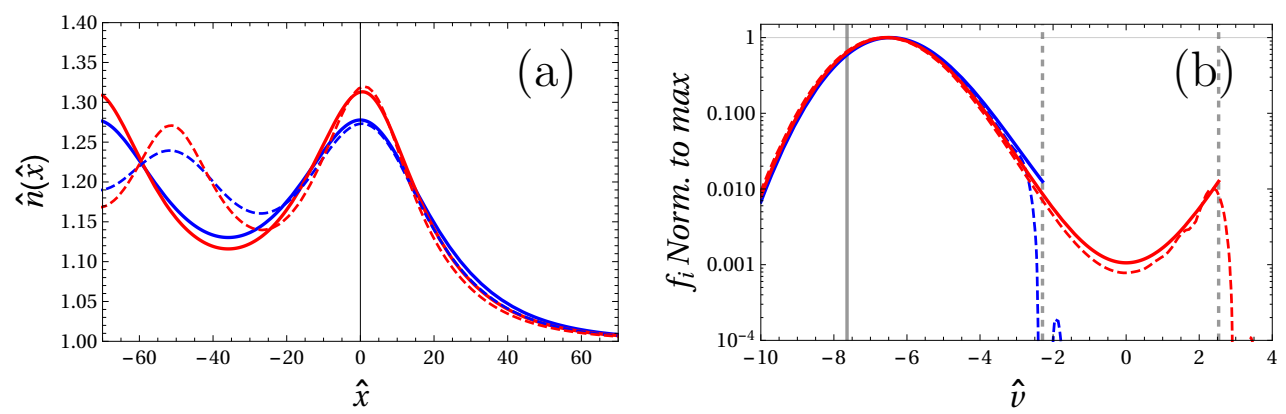

FIG. 3: Comparison of analytical and simulation results. (a) Relative density difference from the far upstream value. Red: ion; blue: electron. Thick solid lines: analytical model; dashed lines: simulation at $t \omega_{p p}=30 . \hat{x}$ denotes the normalized length from the top of the shock. (b) The ion distribution function $f_{i}$ normalized to its maximum value in the upstream $(\hat{x}=15$, red $)$ and downstream $(\hat{x}=-15$, blue) regions. Thick solid lines: analytical; dashed lines: simulation result at $t \omega_{p p}=30$. Solid vertical bar marks the far upstream ion flow speed in shock frame $(-\hat{V})$, and dotted vertical bars correspond to $\hat{v}_{-}$ and $\hat{v}_{+}$.

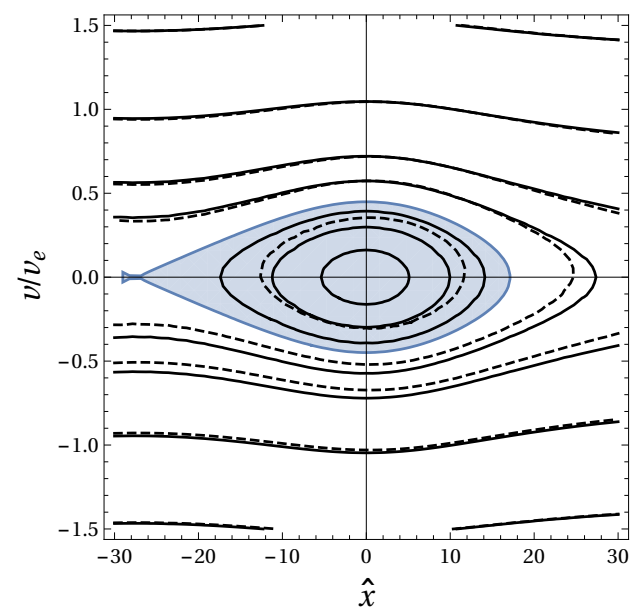

FIG. 4: Contours of the electron distribution function, normalized such that $\tilde{f}_{e}\left(\hat{x}, v / v_{e}=0\right)=\hat{n}_{e}(\hat{x})$. Solid lines: assuming Maxwell-Boltzmann electron distribution; dashed lines: simulation result. The lines show $\tilde{f}_{e}\left(\hat{x}, v / v_{e}\right)=\{0.4,0.75,1,1.2,1.24,1.28\}$, converging about the origin in this order (note that for the simulation results, the 1.24 and 1.28 contours do not exist). Blue shaded area shows the trapped region in the electron phase space.

Finally, we consider the electron distribution, which in the analytical model is taken to be Maxwell-Boltzmann. However, in reality, electrons can get trapped around potential maxima, and there they will develop deviations from a Boltzmann response. This situation indeed happens in the simulation, as illustrated in Figs. 4 and 5 . The trapped region in the phase space is illustrated by the blue shaded area in Fig. 4. where the kinetic energy of electrons is lower than the potential difference between $\hat{\phi}_{\max }$, and $\hat{\phi}_{\min ,-1}$, the first potential minimum downstream. When we compare the Maxwell-Boltzmann model (solid lines), and the simulation results (dashed), we find that they are close at high electron speeds, where the potential can only slightly perturb the total energy of the electrons. However, at lower speeds the simulations show increasing deviations from the Maxwell-Boltzmann response. In particular, the distribution inside the trapped region is much flatter (thus the 


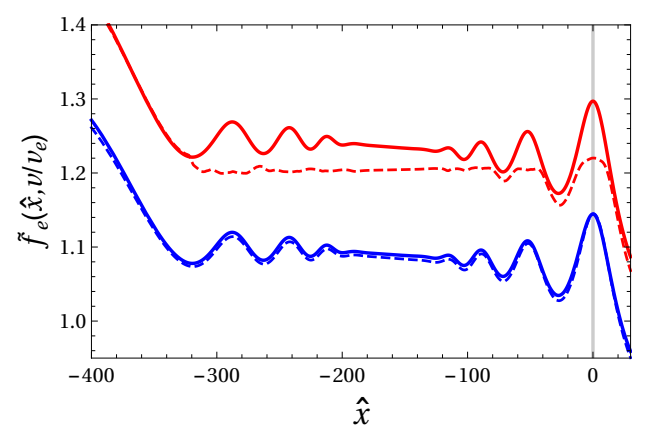

FIG. 5: Electron distribution function, normalized such that $\tilde{f}_{e}\left(\hat{x}, v / v_{e}=0\right)=\hat{n}_{e}(\hat{x})$. Solid lines: assuming Maxwell-Boltzmann electron response; dashed lines: simulation result. Red: $\tilde{f}_{e}\left(\hat{x}, v / v_{e}=0\right)$; blue: $\tilde{f}_{e}\left(\hat{x}, v / v_{e}=+0.5\right)$.

two highest contours do not exist for the simulated distribution). When we take a velocity $\left|v / v_{e}\right|>0.45$, where no trapped region exists, we see a remarkable agreement between the analytical and simulated electron distribution functions, as shown by the blue lines in Fig. $5\left(v / v_{e}=+0.5\right)$, while in all the trapped regions about the local $\hat{\phi}$ maxima, we find a flattening of the distribution, and a reduced electron density, accordingly, see the red lines taken at $v / v_{e}=0$.

\section{SHOCK SOLUTIONS AND REFLECTED IONS}

The Vlasov-Poisson simulations presented in the previous section confirm that the semianalytical model captures the main properties of the shock structure. We therefore proceed to use the model to study the shock solutions, in particular the effect of impurities and electron-to-ion temperature ratio.

To determine the maximum electrostatic potential, we solve $\Phi\left(\hat{\phi}_{\max }, \hat{\phi}_{\max }\right)=0$, with input parameters $\tau$ and $\hat{V}$. As it was pointed out in Ref. 19, not all combinations of $\tau$ and $\hat{V}$ give solutions. In particular, numerical results indicate that $\tau$ needs to exceed a specific value for the existence of solutions. As in Ref.19, for a single ion species (protons), we have a solution for $\hat{V}=4.5$ and $\tau=15$ (corresponding to $M=1.16$ ) with a maximum normalized electrostatic potential $\hat{\phi}_{\max }=1.29$ and a correspondingly reflected fraction $\alpha_{i}=0.0019$.

We start by adding a small amount of impurities to the pure proton plasma. Already, the addition of only $1 \%$ of a fully ionized carbon species $\left(n_{z} / n_{i}=0.01\right)$ changes the solutions considerably. The maximum normalized electrostatic potential increases to $\hat{\phi}_{\max }=1.676$, and consequently, the reflected main ion fraction doubles to $\alpha_{i}=0.0038$. As the fraction of carbon impurities increases, $\hat{\phi}_{\max }$ continues to increase; however, for more than $9 \%$ carbon, there exists no solutions for the shock velocity $\hat{V}=4.5$ and temperature ratio $\tau=15$.

The existence of a shock solution is also affected by the ionization degree. We find that for singly ionized carbon, solutions exist even for very high concentrations, up to $30 \%$. Figure 6 a shows the electrostatic potential for $\hat{V}=4.5$ and $\tau=15$ in the case of a pure hydrogen plasma (solid) and a hydrogen plasma contaminated by $1 \%$ of fully ionized carbon (dashed). Figure $6 \mathrm{~b}$ shows the dependence of the maximum normalized electrostatic potential for three types of impurities on the impurity fraction. We find that for a given impurity, $\hat{\phi}_{\max }$ increases approximately linearly with impurity concentration. In addition, $\hat{\phi}_{\max }$ increases more rapidly with concentration for impurities that have a higher charge, however the slope is not simply proportional to the charge number of the impurity (which is evident from comparing $\hat{\phi}_{\max }$ for $1 \% \mathrm{C}^{6+}$ with $6 \% \mathrm{C}^{1+}$ ).

It is instructive to investigate how the solutions change if the temperature ratio $\tau$ is assumed to be larger. Higher $\tau$ is relevant for high-intensity laser-driven ion acceleration 

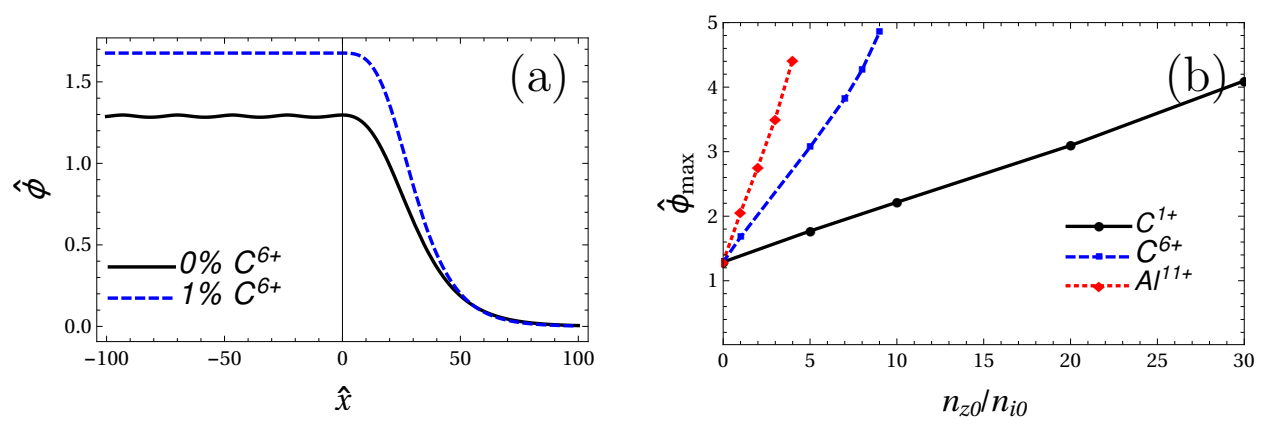

FIG. 6: (a) Electrostatic potential for $\hat{V}=4.5$ and $\tau=15$ for the case of a pure proton plasma (solid) and a $1 \%$ of fully ionized carbon (dashed). (b) Maximum normalized electrostatic potential for various fractions of impurities for $\hat{V}=4.5$ and $\tau=15$.
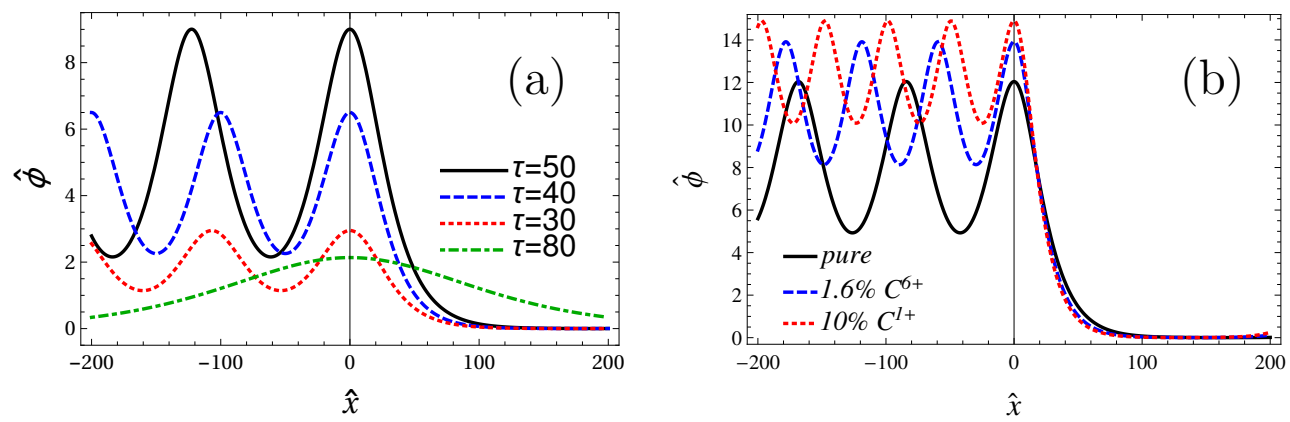

FIG. 7: (a) Electrostatic potential for various temperature ratios $\tau$, for the case of a pure proton plasma and Mach number close to unity. Solid is $\hat{V}=7.8$ and $\tau=50$, blue dashed is for $\hat{V}=7$ and $\tau=40$, red dotted is for $\hat{V}=6$ and $\tau=30$, green dash-dotted is for $\hat{V}=9.2$ and $\tau=80$. (b) Electrostatic potential for $\hat{V}=8$ and $\tau=50$, for the case of a pure proton plasma (solid), $1.6 \%$ of fully ionized carbon (blue dashed) and $10 \%$ of singly ionized carbon (red dotted).

experiments as the laser mostly heats the electrons. Note that the Mach number is reduced with growing $\tau$, but the reflected ion fraction, which involves only $\hat{V}$ and $\hat{\phi}_{\max }$, will not be directly affected by the change in $\tau$, only indirectly. In agreement with Ref. 20, we find that for higher $\tau$ the range of possible Mach numbers is wider.

Figure 7(a) shows the electrostatic potential for various values of $\tau$. The maximum value increases with $\tau$ until a certain limit is reached (at around $\tau=60$ ) and above this value, the solutions become symmetric and soliton-like. The presence of impurities increases the maximum electrostatic potential and thereby the reflected ion fraction. A similar effect results from the presence of $1.6 \%$ of fully ionized carbon as $10 \%$ of singly ionized carbon, as shown in Figure 7(b).

We find that the reflected ion fraction grows with Mach number for fixed electron-to-ion temperature ratio. Figure $8 \mathrm{a}$ shows the growth of the reflected ion fraction for various fractions of fully ionized carbon in a hydrogen plasma. Note that a small change in the Mach number can lead to several orders of magnitude of change in the reflected ion fraction. Figure $8 \mathrm{~b}$ shows that the difference between the maximum and minimum of the electrostatic potential in the downstream region is smaller with increasing impurity concentration, and at a certain shock speed, solutions cease to exist. This maximum shock speed decreases with impurity concentration.

It is interesting to point out that hydrogen has a charge to mass ratio $Z_{i} / \hat{m}_{i}=1$, that is approximately twice as large as that of any other fully charged ion species. This difference leads to a strong disparity between the behavior of hydrogen as main ion or as 

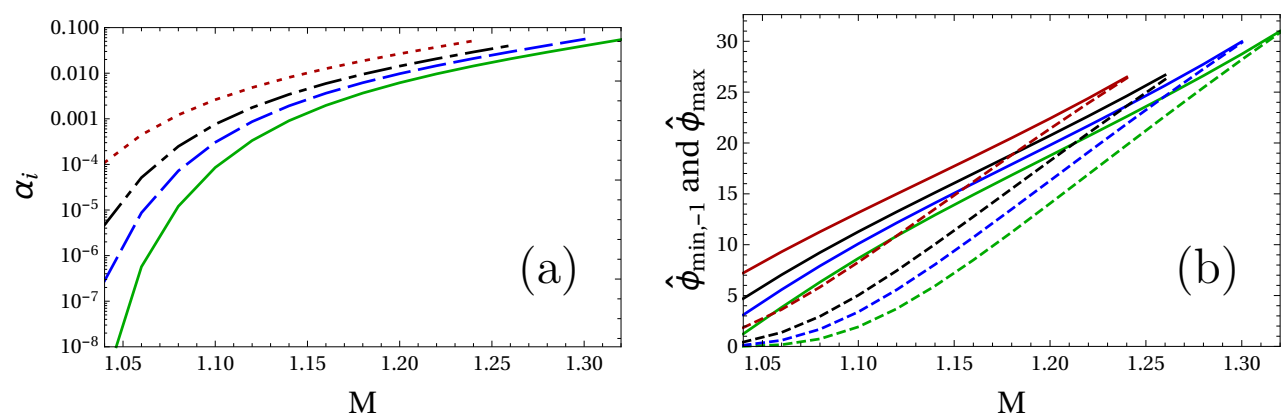

FIG. 8: (a) Reflected ion fraction as function of Mach number for various concentrations of fully ionized carbon in a hydrogen plasma. Solid (green) line is for pure plasma, dashed (blue) is for $1 \%$ carbon, dash-dotted (black) is for $2 \%$ carbon and dotted (dark red) is for $4 \%$ carbon. The electron-to-ion temperature ratio is $\tau=50$. (b) Maximum (solid) and minimum (dashed) of the electrostatic potential in the downstream region for the impurity concentrations given in (a). The colors represent different concentrations of fully ionized carbon in a hydrogen plasma. Green, blue, black, and dark-red correspond to $0 \%, 1 \%, 2 \%$, and $4 \%$, respectively.

impurity, which we would like to illuminate mathematically in the following. Besides $\tau \gg 1$, we only assume that the impurity concentration is sufficiently small for the propagation speed of the shock to be determined by the main ions. This assumption means that $\hat{V}=$ $M \sqrt{Z_{i} \tau / \hat{m}_{i}}$ with some $M \gtrsim 1$. We can express Eq. 44 in terms of $M$ by using the previous expression together with $\hat{\phi}_{\max }=F M^{2} \tau / 2$, where the constant $F$ can be determined by solving $\Phi\left(\hat{\phi}_{\max }, \hat{\phi}_{\max }\right)=0$. Furthermore, for large $\tau$, the $\operatorname{erf}\left(\tilde{V}_{j}\right)$ terms in Eq. (4) for $j=i$ can be very well approximated by 1 , and recalling that $\hat{T}_{i}=1$, we find that the reflected fraction of main ions is

$$
\alpha_{i}=\frac{1}{2}\left\{1-\operatorname{erf}\left[(1-\sqrt{F}) M \sqrt{\frac{Z_{i} \tau}{2}}\right]\right\} .
$$

Now it is apparent that $F=1$ gives $\alpha_{i}=1 / 2$, and $\alpha_{i}$ decreases rapidly as $F$ gets smaller than 1. In particular, if $1-\sqrt{F}$ becomes larger than $\sqrt{2 /\left(Z_{i} \tau\right)} \ll 1, \alpha_{i}$ is very small. In practice, this result sets $\hat{\phi}_{\max }$ to be slightly smaller than $M^{2} \tau / 2$ for physically interesting cases (i.e., where $\alpha_{j}$ is not vanishingly small). Using the above procedure, we find the following expression for the reflected fraction of impurities

$$
\alpha_{z}=\frac{1}{2}\left\{1+\operatorname{erf}\left[M \sqrt{\frac{F Z_{z} \tau}{2 \hat{T}_{z}}}\left(1-\sqrt{\frac{\hat{m}_{z} Z_{i}}{F \hat{m}_{i} Z_{z}}}\right)\right]\right\} .
$$

Since $F \approx 1$, and for hydrogen main species $\hat{m}_{z} Z_{i} /\left(\hat{m}_{i} Z_{z}\right)>1$, the term in the parentheses is a negative number, and $\alpha_{z}$ evaluates to an extremely small number. However, for hydrogen impurity the parenthetical term is positive, and $\alpha_{z}$ is close to unity, although $1-\alpha_{z}$ is not necessarily extremely small. In other words, in a hydrogen plasma, the impurities are practically unreflected by the shock. However, in a non-hydrogenic plasma almost all hydrogen impurities are reflected. The latter conclusion is illustrated in the following through a numerical simulation.

We performed a Gkeyll simulation for a fully ionized aluminum plasma $\left(Z_{i}=13, \hat{m}_{i}=\right.$ 27 ) with hydrogen impurity $\left(Z_{z}=1, \hat{m}_{z}=1\right)$ of concentration $n_{z} / n_{i}=0.01$, and equal ion temperatures $T_{i}=T_{z}$. The normalized electron temperature is $\tau=45$, and the simulation is initiated with a density discontinuity with a density ratio 2 . The simulation used similar resolution parameters to those in the simulation shown in Sec. [II] except that the range of 

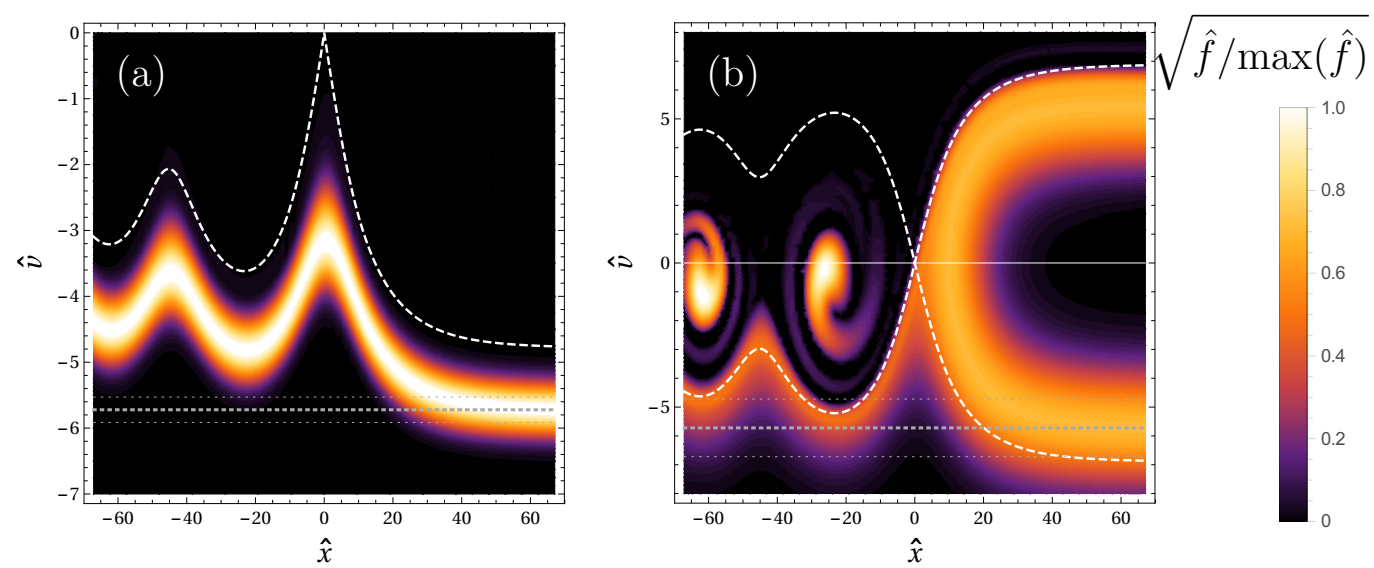

FIG. 9: Distribution functions of the aluminum main ion species (a), and the hydrogen impurity (b), from a Gkeyll simulation. Dashed lines correspond to the separatrices, $\hat{v}_{ \pm}(\hat{x})$. The far-upstream ion flow speed in the shock frame, $-\hat{V}$, is shown with thick dotted line, and one thermal speed width of the distribution is indicated by the thin dotted lines $-\hat{V} \pm v_{j}$. Mind the different $\hat{v}$ scales.

velocities is $\left\{-18 v_{i}, 54 v_{i}\right\}$ for aluminum and $\left\{-6 v_{z}, 18 v_{z}\right\}$ for hydrogen, and the number of cells in velocity space is 96 .

We consider the solution at $t \sqrt{m_{e} / m_{p}} \omega_{p e}=35$, where $\omega_{p e}=\sqrt{e^{2} n_{e 0} /\left(\epsilon_{0} m_{e}\right)}$, with $n_{e 0}$ the far upstream electron density. In the simulation we find that the shock potential is $\hat{\phi}_{\max }=24.09$, and the shock propagates with a speed $\hat{V}=5.72$, corresponding to $M=$ 1.229. According to $\hat{\phi}_{\max }=F M^{2} \tau / 2$, this set of parameters translates to $F=0.709$, which is rather low, suggesting that the reflected main ion fraction is small. Indeed, from Eq. (8) we estimate $\alpha_{i}=1.33 \cdot 10^{-6}$, and in the simulation $\alpha_{i}$ is so small that it cannot be meaningfully evaluated within the finite numerical accuracy. However, as expected, the ratio of reflected hydrogen impurities is substantial: from Eq. (9) we estimate $\alpha_{z}=0.889$, and taking the ratio of the densities in the incoming and reflected populations at $\hat{x}=62.6$ yields a comparable $\alpha_{z} \approx 0.874$. Finally, we note that for the same $\tau$ and $\hat{V}$ the semianalytical calculation gives a somewhat higher shock potential $\hat{\phi}_{\max }=25.953(F=0.763)$ that corresponds to $\alpha_{i}=8.69 \cdot 10^{-5}$ (note the sensitivity to $F$ ) and $\alpha_{z} \approx 0.93$.

Sections of the simulated distribution functions of the two ion species around the shock front are shown in Fig. 9. In addition, the separatrices $\hat{v}=\hat{v}_{ \pm}(\hat{x}) \equiv \pm \sqrt{2\left(Z_{j} / \hat{m}_{j}\right)\left[\hat{\phi}_{\max }-\hat{\phi}(\hat{x})\right]}$, with $\hat{\phi}(\hat{x})$ also taken from the simulation, are shown with dashed lines. The far upstream flow speed $-\hat{V}$, together with species thermal speed deviations from it, $-\hat{V} \pm v_{j}$, are indicated by the thick and thin dotted lines, respectively. For aluminum, shown in Fig. 9a, we can see that the maximum of the far upstream ion distribution is several thermal speeds below the separatrix, which is consistent with the low reflected ion fraction. For hydrogen, shown in Fig. 9b, a major part of the distribution is above the separatrix, hence the large reflected fraction. In contrast to the pure hydrogen plasma case shown earlier, and the aluminum main species, the phase space of the hydrogen impurity is not empty behind the shock, above the separatrix: blobs of hydrogen are being trapped in these regions. Interestingly $f_{z}$ reaches twice as high values in the trapped region than upstream, suggesting that they cannot originate from the upstream region. In fact, the blobs are torn away from the large density region of the initial density discontinuity, and are now trapped by the downstream oscillations of the potential.

For comparison, in Fig. 10 we also show the ion distribution in the pure hydrogen simulation of Sec. III] where most of the far-upstream distribution is below the separatrix, thus only a small fraction of ions is reflected. 


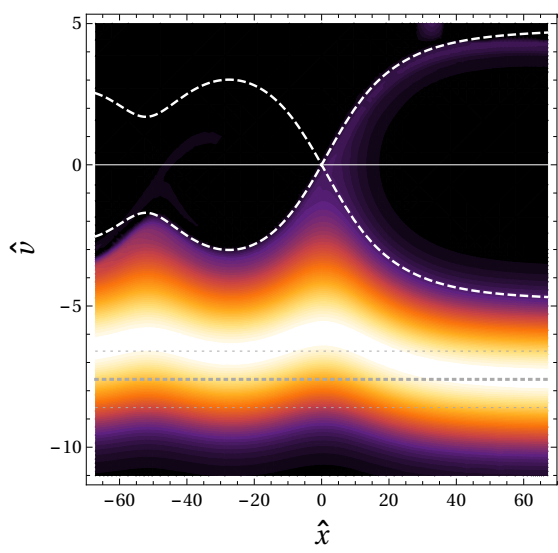

FIG. 10: Ion distribution function from a pure hydrogen plasma simulation. Dashed lines correspond to the separatrices, $\hat{v}_{ \pm}(\hat{x})$. The far-upstream ion flow speed in the shock frame, $-\hat{V}$, is shown with thick dotted line, and one thermal speed width of the distribution is indicated by the thin dotted lines $-\hat{V} \pm v_{j}$.

\section{v. DISCUSSION AND CONCLUSIONS}

In this paper, we extend the analysis of low-Mach number electrostatic shock structures with a semi-analytical model. We assume Maxwell-Boltzmann electrons and an ion distribution that is extended along contours of constant total energy from a Maxwellian far upstream. The self-consistent electrostatic field is calculated using Poisson's equation. Regarding these aspects, it is similar to the model of Cairns et a $\sqrt{1920}$. However, in the model described in Refs. 19 and 20, the ion distribution in the downstream region is finite below $v=0$ in the shock frame, and zero above, which appears to be inconsistent in a steady state shock model (except in monotonic shocks without trapped regions downstream). The difference between the models affects only the downstream properties of the shock but not its existence or the reflected ion fractions.

We compare the semi-analytical model to Eulerian Vlasov-Poisson simulations with Gkeyll, where we consider shocks generated by the decay of an initial density discontinuity. We find that the model well reproduces the simulated shock potential and Mach number. The electron distribution function is well approximated by a Maxwell-Boltzmann distribution in most of the phase space, while simulations show signatures of trapping in the downstream oscillations of the potential, depleting the low speed population of electrons around the local potential maxima. In single species and hydrogen bulk simulations the ion distribution is well captured by our ansatz; in particular, the downstream trapped regions of the ion phase space are empty. However, for non-hydrogenic main species, trapping of hydrogen impurities can occur.

We used the semi-analytical model to study the effect of heavy ion impurities on the shock parameters and reflected ion fraction. This study is of relevance to laser-based ion acceleration experiments, which are rarely free of impurities, for example. We find, that only a few percent of fully ionized carbon impurity in a hydrogen plasma will give a large effect on the reflected ion fraction. This is because the maximum electrostatic potential grows considerably. However, the maximum reflected fraction of hydrogen ions remains below $10 \%$ and the Mach number is low, therefore these shocks are not expected to be efficient accelerators of a large number of ions to very high energies, rather their strength lies in their mono-energeticity.

We provide accurate analytical expressions for the reflected fractions of main ions and impurities, which illuminate the different behavior of hydrogen, depending on its role as main ion or impurity. In a hydrogen plasma with a large electron-to-ion temperature ratio, the reflection of non-hydrogenic impurities is vanishingly small. On the other hand, one 
way to increase the reflected fraction of hydrogen ions is to create a shock in a heavy ion plasma containing hydrogen impurities, in which case almost all of the hydrogen will be reflected, with a speed close to twice the shock speed. Similarly, the different behavior of hydrogen as a main species or as an impurity has been reported before in the context of the expansion of a multi-species plasma into vacuum $34[35$.

\section{ACKNOWLEDGMENTS}

The authors would like to thank A Stahl for numerical advice, together with L Gremillet, E Siminos, T C DuBois, and A Sundström for useful comments on the manuscript. This work was supported by the International Career Grant (Dnr. 330-2014-6313) from Vetenskapsrådet, and Marie Sklodowska Curie Actions, Cofund, Project INCA 600398; the European Research Council (ERC-2014-CoG grant 647121), the Knut and Alice Wallenberg Foundation, and the National Science Foundation (NSF) SHINE award No. AGS-1622306. This development of Gkeyll code is partly funded by the U.S. Department of Energy under Contract No. DE-AC02-09CH11466 and by the Air Force Office of Scientific Research under grant number FA9550-15-1-0193. This work used the Extreme Science and Engineering Discovery Environment (XSEDE), which is supported by NSF grant number ACI-1548562.

${ }^{1}$ D. A. Tidman and N. A. Krall, Shock Waves in Collisionless Plasmas (Wiley series in plasma physics) (Wiley-Interscience, 1971).

${ }^{2}$ A. Marcowith, A. Bret, A. Bykov, M. E. Dieckman, L. O. Drury, B. Lembge, M. Lemoine, G. Morlino, G. Murphy, G. Pelletier, I. Plotnikov, B. Reville, M. Riquelme, L. Sironi, and A. S. Novo, "The microphysics of collisionless shock waves," Reports on Progress in Physics 79, 046901 (2016)

${ }^{3}$ D. Haberberger, S. Tochitsky, F. Fiuza, C. Gong, R. A. Fonseca, L. O. Silva, W. B. Mori, and C. Joshi, "Collisionless shocks in laser-produced plasma generate monoenergetic high-energy proton beams," Nat Phys 8, 95-99 (2012)

${ }^{4}$ F. Fiuza, A. Stockem, E. Boella, R. A. Fonseca, L. O. Silva, D. Haberberger, S. Tochitsky, W. B. Mori, and C. Joshi, "Ion acceleration from laser-driven electrostatic shocks," Physics of Plasmas 20, 056304 (2013) http://dx.doi.org/10.1063/1.4801526

${ }^{5}$ B. S. Wettervik, T. C. DuBois, and T. Flp, "Vlasov modelling of laser-driven collisionless shock acceleration of protons," Physics of Plasmas 23, 053103 (2016), http://dx.doi.org/10.1063/1.4948424

${ }^{6}$ R. J. Taylor, D. R. Baker, and H. Ikezi, "Observation of collisionless electrostatic shocks," Phys. Rev. Lett. 24, 206-209 (1970)

${ }^{7}$ L. Romagnani, S. V. Bulanov, M. Borghesi, P. Audebert, J. C. Gauthier, K. Löwenbrück, A. J. Mackinnon, P. Patel, G. Pretzler, T. Toncian, and O. Willi, "Observation of collisionless shocks in laser-plasma experiments," Phys. Rev. Lett. 101, 025004 (2008)

${ }^{8}$ T. Morita, Y. Sakawa, Y. Kuramitsu, S. Dono, H. Aoki, H. Tanji, T. N. Kato, Y. T. Li, Y. Zhang, X. Liu, J. Y. Zhong, H. Takabe, and J. Zhang, "Collisionless shock generation in highspeed counterstreaming plasma flows by a high-power laser," Physics of Plasmas 17, 122702 (2010) http://dx.doi.org/10.1063/1.3524269

${ }^{9}$ F. S. Mozer, C. W. Carlson, M. K. Hudson, R. B. Torbert, B. Parady, J. Yatteau, and M. C. Kelley, "Observations of paired electrostatic shocks in the polar magnetosphere," Phys. Rev. Lett. 38, 292-295 (1977)

${ }^{10}$ R. Z. Sagdeev, "Cooperative Phenomena and Shock Waves in Collisionless Plasmas," Reviews of Plasma Physics 4, 23 (1966).

${ }^{11}$ S. S. Moiseev and R. Z. Sagdeev, "Collisionless shock waves in a plasma in a weak magnetic field," Journal of Nuclear Energy. Part C, Plasma Physics, Accelerators, Thermonuclear Research 5, 43 (1963)

${ }^{12}$ D. W. Forslund and C. R. Shonk, "Formation and structure of electrostatic collisionless shocks," Phys. Rev. Lett. 25, 1699-1702 (1970)

${ }^{13}$ M. E. Dieckmann, G. Sarri, L. Romagnani, I. Kourakis, and M. Borghesi, "Simulation of a collisionless planar electrostatic shock in a protonelectron plasma with a strong initial thermal pressure change," Plasma Physics and Controlled Fusion 52, 025001 (2010)

${ }^{14}$ G. Sarri, M. E. Dieckmann, I. Kourakis, and M. Borghesi, "Shock creation and particle acceleration driven by plasma expansion into a rarefied medium," Physics of Plasmas 17, 082305 (2010) https://doi.org/10.1063/1.3469762

${ }^{15}$ A. Macchi, A. S. Nindrayog, and F. Pegoraro, "Solitary versus shock wave acceleration in laser-plasma interactions," Phys. Rev. E 85, 046402 (2012)

${ }^{16} \mathrm{C}$. Birdsall and A. Langdon, Plasma Physics via Computer Simulation (Series in Plasma Physics) (CRC Press, 2004).

${ }^{17}$ A. Grassi, L. Fedeli, A. Sgattoni, and A. Macchi, "Vlasov simulation of laser-driven shock acceleration and ion turbulence," Plasma Physics and Controlled Fusion 58, 034021 (2016) 
${ }^{18}$ A. Smith, "An exact electrostatic shock solution for a collisionless plasma," Journal of Plasma Physics 4, $549561(1970)$

${ }^{19}$ R. A. Cairns, R. Bingham, P. Norreys, and R. Trines, "Laminar shocks in high power laser plasma interactions," Physics of Plasmas 21, 022112 (2014) http://dx.doi.org/10.1063/1.4864328

${ }^{20}$ R. A. Cairns, R. Bingham, R. G. M. Trines, and P. Norreys, "Weak collisionless shocks in laser-plasmas," Plasma Physics and Controlled Fusion 57, 044008 (2015)

${ }^{21}$ J. Juno, A. Hakim, J. TenBarge, E. Shi, and W. Dorland, "Discontinuous galerkin algorithms for fully kinetic plasmas," Journal of Computational Physics 353, 110 - 147 (2018)

${ }^{22}$ M. A. Malkov, R. Z. Sagdeev, G. I. Dudnikova, T. V. Liseykina, P. H. Diamond, K. Papadopoulos, C.-S. Liu, and J. J. Su, "Ion-acoustic shocks with self-regulated ion reflection and acceleration," Physics of Plasmas 23, 043105 (2016) http://dx.doi.org/10.1063/1.4945649

${ }^{23}$ H. Schamel, "Stationary solitary, snoidal and sinusoidal ion acoustic waves," Plasma Physics 14, 905 (1972)

${ }^{24}$ G. I. Dudnikova and A. A. Efimova, "Numerical models of the ion-acoustic collisionless shock," AIP Conference Proceedings 1773, 090001 (2016) http://aip.scitation.org/doi/pdf/10.1063/1.4964992

${ }^{25}$ A. V. Gurevich, "Distribution of captured particles in a potential well in the absence of collisions," Soviet Physics JETP 26, 575 (1968)

${ }^{26}$ S. C. Wilks and W. L. Kruer, "Absorption of ultrashort, ultra-intense laser light by solids and overdense plasmas," IEEE Journal of Quantum Electronics 33, 1954-1968 (1997)

${ }^{27}$ B. D. Fried, R. B. White, and T. K. Samec, "Ion acoustic waves in a multiion plasma," The Physics of Fluids 14, 2388-2392 (1971), http://aip.scitation.org/doi/pdf/10.1063/1.1693346.

${ }^{28}$ B. Eliasson, "Ion shock acceleration by large amplitude slow ion acoustic double layers in laser-produced plasmas," Physics of Plasmas 21, 023111 (2014) http://dx.doi.org/10.1063/1.4866240

${ }^{29}$ T. N. Kato and H. Takabe, "Electrostatic and electromagnetic instabilities associated with electrostatic shocks: Two-dimensional particle-in-cell simulation," Physics of Plasmas 17, 032114 (2010) https://doi.org/10.1063/1.3372138

${ }^{30}$ M. E. Dieckmann, G. Sarri, D. Doria, M. Pohl, and M. Borghesi, "Modification of the formation of high-mach number electrostatic shock-like structures by the ion acoustic instability," Physics of Plasmas 20, 102112 (2013) https://doi.org/10.1063/1.4825339.

${ }^{31}$ A. Stockem, T. Grismayer, R. A. Fonseca, and L. O. Silva, "Electromagnetic field generation in the downstream of electrostatic shocks due to electron trapping," Phys. Rev. Lett. 113, 105002 (2014)

${ }^{32}$ T. D. Arber, K. Bennett, C. S. Brady, A. Lawrence-Douglas, M. G. Ramsay, N. J. Sircombe, P. Gillies, R. G. Evans, H. Schmitz, A. R. Bell, and C. P. Ridgers, "Contemporary particle-in-cell approach to laser-plasma modelling," Plasma Physics and Controlled Fusion 57, 113001 (2015)

${ }^{33}$ R. J. Mason, "Structure of evolving ionacoustic fronts in collisionless plasmas," The Physics of Fluids 13, 1042-1048 (1970), http://aip.scitation.org/doi/pdf/10.1063/1.1693006

${ }^{34}$ V. T. Tikhonchuk, A. A. Andreev, S. G. Bochkarev, and V. Y. Bychenkov, "Ion acceleration in shortlaser-pulse interaction with solid foils," Plasma Physics and Controlled Fusion 47, B869 (2005)

${ }^{35}$ A. P. L. Robinson, A. R. Bell, and R. J. Kingham, "Effect of target composition on proton energy spectra in ultraintense laser-solid interactions," Phys. Rev. Lett. 96, 035005 (2006) 